\title{
Schur-Convexity for a Class of Completely Symmetric Function Dual
}

\author{
Huan-Nan Shi ${ }^{\mathrm{a}}$, Wei-Shih Du ${ }^{\mathrm{b}}$ \\ ${ }^{a}$ Department of Electronic Information, Teacher's College, Beijing Union University, Beijing 100011, P. R. China \\ ${ }^{b}$ Department of Mathematics, National Kaohsiung Normal University, Kaohsiung 82444, Taiwan
}

\begin{abstract}
By using the decision theorem and properties of the Schur-convex function, the Schur-geometric convex function and the Schur-harmonic function, the Schur- convexity, Schur-geometric convexity and Schurharmonic convexity of a class of complete symmetric functions are studied. As applications, some symmetric function inequalities are established.
\end{abstract}

Keywords: Schur-convexity; Schur-geometric convexity; Schur-harmonic convexity; completely symmetric function; dual form.

2010 MSC: 05E05, 26B25.

\section{Introduction}

Let us begin with some basic definitions and notation that will be needed in this paper. Throughout this paper, we denote by $\mathbb{N}$ and $\mathbb{R}$, the set of positive integers and real numbers, respectively. Denote

$$
\begin{gathered}
\mathbb{R}^{n}:=\left\{\boldsymbol{x}=\left(x_{1}, x_{2}, \cdots, x_{n}\right): x_{i} \in \mathbb{R}, i=1,2, \ldots, n\right\}, \\
\mathbb{R}_{+}^{n}:=\left\{\boldsymbol{x}=\left(x_{1}, x_{2}, \ldots, x_{n}\right): x_{i}>0, i=1,2, \ldots, n\right\}
\end{gathered}
$$

and

$$
\mathbb{R}_{-}^{n}:=\left\{\boldsymbol{x}=\left(x_{1}, x_{2}, \ldots, x_{n}\right): x_{i}<0, i=1,2, \ldots, n\right\},
$$

where $n \in \mathbb{N}$. In particular, we simply use the notations $\mathbb{R}$ and $\mathbb{R}_{+}$instead of $\mathbb{R}^{1}$ and $\mathbb{R}_{+}^{1}$, respectively.

Email addresses: shihuannan2014@qq.com (Huan-Nan Shi), wsdu@mail.nknu.edu.tw (Wei-Shih Du) 
During the past more than two decades, many authors are dedicated to the hot topic of inequality research area on the Schur-convexity, Schur-geometric and Schur-harmonic convexity of various symmetric functions; see, e.g., [7]-[25] and references therein.

The family of complete symmetric functions is an important class of symmetric functions.

For $\boldsymbol{x}=\left(x_{1}, x_{2}, \ldots, x_{n}\right) \in \mathbb{R}^{n}$, the complete symmetric function $c_{n}(\boldsymbol{x}, r)$ is defined by

$$
c_{n}(\boldsymbol{x}, r)=\sum_{i_{1}+i_{2}+\cdots+i_{n}=r} x_{1}^{i_{1}} x_{2}^{i_{2}} \cdots x_{n}^{i_{n}}
$$

where $c_{0}(\boldsymbol{x}, r)=1, r \in\{1,2, \ldots, n\}, \quad i_{1}, i_{2}, \ldots, i_{n}$ are non-negative integers.

Guan [11] discussed the Schur-convexity of $c_{n}(\boldsymbol{x}, r)$ and proved the following proposition.

Proposition 1. $c_{n}(\boldsymbol{x}, r)$ is increasing and Schur-convex on $\mathbb{R}_{+}^{n}$.

Subsequently, Chu et al. [8] prove the following proposition.

Proposition 2. $c_{n}(\boldsymbol{x}, r)$ is Schur-geometrically convex and Schur-harmonically convex on $\mathbb{R}_{+}^{n}$.

The dual form of the complete symmetric function $c_{n}(\boldsymbol{x}, r)$ is defined by

$$
c_{n}^{*}(\boldsymbol{x}, r)=\prod_{i_{1}+i_{2}+\cdots+i_{n}=r} \sum_{j=1}^{n} i_{j} x_{j},
$$

where $c_{0}^{*}(\boldsymbol{x}, r)=1, r \in\{1,2, \ldots, n\}, \quad i_{1}, i_{2}, \ldots, i_{n}$ are non-negative integers.

Zhang and Shi 24] established the following two propositions.

Proposition 3. For $r=1,2, \ldots, n, c_{n}^{*}(\boldsymbol{x}, r)$ is increasing and Schur-concave on $\mathbb{R}_{+}^{n}$.

Proposition 4. For $r=1,2, \ldots, n, c_{n}^{*}(\boldsymbol{x}, r)$ is Schur-geometrically convex and Schur-harmonically convex on $\mathbb{R}_{+}^{n}$.

Notice that

$$
c_{n}^{*}(-\boldsymbol{x}, r)=(-1)^{r} c_{n}^{*}(\boldsymbol{x}, r) .
$$

It is not difficult to verify the following proposition.

Proposition 5. If $r$ is even integer ( or odd integer, respectively), then $c_{n}^{*}(\boldsymbol{x}, r)$ is decreasing and Schurconcave ( or increasing and Schur-convex, respectively ) on $\mathbb{R}_{-}^{n}$.

In 2014, Sun et al. [12] studied the Schur-convexity, Schur-geometric and harmonic convexities of the following composite function of $c_{n}(\boldsymbol{x}, r)$

$$
c_{n}\left(\frac{\boldsymbol{x}}{1-\boldsymbol{x}}, r\right)=\sum_{i_{1}+i_{2}+\cdots+i_{n}=r} \prod_{j=1}^{n}\left(\frac{x_{j}}{1-x_{j}}\right)^{i_{j}} .
$$

Using Lemmas 1, 2 and 3 in second section, they proved the following Theorems A, B and C, respectively.

Theorem A. For $\boldsymbol{x}=\left(x_{1}, x_{2}, \ldots, x_{n}\right) \in(0,1)^{n} \cup(1,+\infty)^{n}$ and $r \in \mathbb{N}$,

(i) $c_{n}\left(\frac{\boldsymbol{x}}{1-\boldsymbol{x}}, r\right)$ is increasing and Schur-convex on $(0,1)^{n}$; 
(ii) if $r$ is even integer (or odd integer, respectively), then $c_{n}\left(\frac{\boldsymbol{x}}{1-\boldsymbol{x}}, r\right)$ is Schur-convex (or Schur-concave, respectively) on $(1,+\infty)^{n}$, and is decreasing (or increasing, respectively).

Theorem B. For $\boldsymbol{x}=\left(x_{1}, x_{2}, \ldots, x_{n}\right) \in(0,1)^{n} \cup(1,+\infty)^{n}$ and $r \in \mathbb{N}$,

(i) $c_{n}\left(\frac{x}{1-x}, r\right)$ is Schur-geometrically convex on $(0,1)^{n}$;

(ii) if $r$ is even integer (or odd integer, respectively), then $c_{n}\left(\frac{\boldsymbol{x}}{1-\boldsymbol{x}}, r\right)$ is Schur-geometrically convex ( or Schur-geometrically concave, respectively) on $(1,+\infty)^{n}$.

Theorem C. For $\boldsymbol{x}=\left(x_{1}, x_{2}, \ldots, x_{n}\right) \in(0,1)^{n} \cup(1,+\infty)^{n}$ and $r \in \mathbb{N}$,

(i) $c_{n}\left(\frac{\boldsymbol{x}}{1-\boldsymbol{x}}, r\right)$ is Schur-harmonically convex on $(0,1)^{n}$;

(ii) if $r$ is even integer (or odd integer, respectively), then $c_{n}\left(\frac{\boldsymbol{x}}{1-\boldsymbol{x}}, r\right)$ is Schur-harmonically convex (or Schur-harmonically concave, respectively) on $(1,+\infty)^{n}$.

In 2016, Shi et al. [25] used the properties of Schur-convex, Schur-geometrically convex and Schurharmonically convex functions respectively to give simple proofs of Theorems A, B and C.

In [25], Shi et al. also further considered the Schur-convexity of $c_{n}(\boldsymbol{x}, r)$ on $\mathbb{R}_{-}^{n}$, which established the following proposition.

Proposition 6. If $r$ is even integer(or odd integer, respectively), then $c_{n}(\boldsymbol{x}, r)$ is decreasing and Schurconvex (or increasing and Schur-concave, respectively) on $\mathbb{R}_{-}^{n}$.

The dual form of the function $c_{n}\left(\frac{x}{1-x}, r\right)$ is defined by

$$
c_{n}^{*}\left(\frac{\boldsymbol{x}}{1-\boldsymbol{x}}, r\right)=\prod_{i_{1}+i_{2}+\cdots+i_{n}=r} \sum_{j=1}^{n} i_{j}\left(\frac{x_{j}}{1-x_{j}}\right) .
$$

A function associated with this function is

$$
c_{n}^{*}\left(\frac{\boldsymbol{x}}{\boldsymbol{x}-1}, r\right)=\prod_{i_{1}+i_{2}+\cdots+i_{n}=r} \sum_{j=1}^{n} i_{j}\left(\frac{x_{j}}{x_{j}-1}\right) .
$$

This paper we will study the Schur-convexity, Schur-geometric and Schur-harmonic convexiies of Symmetric functions $c_{n}^{*}\left(\frac{\boldsymbol{x}}{\boldsymbol{x}-1}, r\right)$ and $c_{n}^{*}\left(\frac{\boldsymbol{x}}{1-\boldsymbol{x}}, r\right)$.

Our main results will be established as follows:

Theorem 1. For $r \in \mathbb{N}, c_{n}^{*}\left(\frac{\boldsymbol{x}}{\boldsymbol{x}-1}, r\right)$ is Schur-convex, Schur-geometrically convex and Schur-harmonically convex on $(1,+\infty)^{n}$.

Theorem 2. For $\boldsymbol{x}=\left(x_{1}, x_{2}, \ldots, x_{n}\right) \in \mathbb{R}_{+}^{n} \cup \mathbb{R}_{-}^{n}$ and $r \in \mathbb{N}$,

(i) $c_{n}^{*}\left(\frac{\boldsymbol{x}}{1-\boldsymbol{x}}, r\right)$ is increasing on $\mathbb{R}_{+}^{n}$ and Schur-convex on $\left[\frac{1}{2}, 1\right)^{n}$;

(ii) if $r$ is even integer (or odd integer, respectively), then $c_{n}^{*}\left(\frac{\boldsymbol{x}}{1-\boldsymbol{x}}, r\right)$ is Schur-convex ( or Schur-concave, respectively) on $(1,+\infty)^{n}$;

(iii) if $r$ is even integer (or odd integer, respectively), then $c_{n}^{*}\left(\frac{\boldsymbol{x}}{1-\boldsymbol{x}}, r\right)$ is decreasing and Schur-concave (or increasing and Schur-convex, respectively) on $\mathbb{R}_{-}^{n}$. 
Theorem 3. For $\boldsymbol{x}=\left(x_{1}, x_{2}, \ldots, x_{n}\right) \in \mathbb{R}_{+}^{n}$ and $r \in \mathbb{N}$,

(i) $c_{n}^{*}\left(\frac{\boldsymbol{x}}{1-\boldsymbol{x}}, r\right)$ is Schur-geometrically convex on $(0,1)^{n}$;

(ii) if $r$ is even integer ( or odd integer, respectively), then $c_{n}^{*}\left(\frac{\boldsymbol{x}}{1-\boldsymbol{x}}, r\right)$ is Schur-geometrically convex ( or Schur-geometrically concave, respectively) on $(1,+\infty)^{n}$.

Theorem 4. For $\boldsymbol{x}=\left(x_{1}, x_{2}, \ldots, x_{n}\right) \in \mathbb{R}_{+}^{n} \cup \mathbb{R}_{-}^{n}$ and $r \in \mathbb{N}$,

(i) $c_{n}^{*}\left(\frac{\boldsymbol{x}}{1-\boldsymbol{x}}, r\right)$ is Schur-harmonically convex on $(0,1)^{n}$;

(ii) if $r$ is even integer ( or odd integer, respectively), then $c_{n}^{*}\left(\frac{\boldsymbol{x}}{1-\boldsymbol{x}}, r\right)$ is Schur-harmonically convex (or Schur-harmonically concave, respectively ) on $(1,+\infty)^{n}$.

\section{Preliminaries}

For convenience, we first recall some known definitions and results.

Definition 1. [1, 2] For $\boldsymbol{x}=\left(x_{1}, x_{2}, \ldots, x_{n}\right)$ and $\boldsymbol{y}=\left(y_{1}, y_{2}, \ldots, y_{n}\right) \in \mathbb{R}^{n}$,

(i) $\boldsymbol{x} \geq \boldsymbol{y}$ means $x_{i} \geq y_{i}$ for all $i=1,2, \ldots, n$.

(ii) Let $\Omega \subset \mathbb{R}^{n}, \varphi: \Omega \rightarrow \mathbb{R}$ is said to be increasing if $\boldsymbol{x} \geq \boldsymbol{y}$ implies $\varphi(\boldsymbol{x}) \geq \varphi(\boldsymbol{y}) . \varphi$ is said to be decreasing if and only if $-\varphi$ is increasing.

Definition 2. [1, 2] For $\boldsymbol{x}=\left(x_{1}, x_{2}, \ldots, x_{n}\right)$ and $\boldsymbol{y}=\left(y_{1}, y_{2}, \ldots, y_{n}\right) \in \mathbb{R}^{n}$,

(i) $\boldsymbol{x}$ is said to be majorized by $\boldsymbol{y}$ (in symbols $\boldsymbol{x} \prec \boldsymbol{y}$ ) if $\sum_{i=1}^{k} x_{[i]} \leq \sum_{i=1}^{k} y_{[i]}$ for $k=1,2, \ldots, n-1$ and $\sum_{i=1}^{n} x_{i}=\sum_{i=1}^{n} y_{i}$, where $x_{[1]} \geq x_{[2]} \geq \cdots \geq x_{[n]}$ and $y_{[1]} \geq y_{[2]} \geq \cdots \geq y_{[n]}$ are rearrangements of $\boldsymbol{x}$ and $\boldsymbol{y}$ in a descending order.

(ii) Let $\Omega \subset \mathbb{R}^{n}, \varphi: \Omega \rightarrow \mathbb{R}$ is said to be a Schur-convex function on $\Omega$ if $\boldsymbol{x} \prec \boldsymbol{y}$ on $\Omega$ implies $\varphi(\boldsymbol{x}) \leq$ $\varphi(\boldsymbol{y}) . \varphi$ is said to be a Schur-concave function on $\Omega$ if and only if $-\varphi$ is Schur-convex function on $\Omega$.

Definition 3. [1, 2] Let $\boldsymbol{x}=\left(x_{1}, x_{2}, \ldots, x_{n}\right)$ and $\boldsymbol{y}=\left(y_{1}, y_{2}, \ldots, y_{n}\right) \in \mathbb{R}^{n}$.

(i) A set $\Omega \subset \mathbb{R}^{n}$ is said to be a convex set if $\boldsymbol{x}, \boldsymbol{y} \in \Omega, 0 \leq \alpha \leq 1$, implies $\alpha \boldsymbol{x}+(1-\alpha) \boldsymbol{y}=$ $\left(\alpha x_{1}+(1-\alpha) y_{1}, \alpha x_{2}+(1-\alpha) y_{2}, \ldots, \alpha x_{n}+(1-\alpha) y_{n}\right) \in \Omega$.

(ii) Let $\Omega \subset \mathbb{R}^{n}$ be convex set. A function $\varphi: \Omega \rightarrow \mathbb{R}$ is said to be a convex function on $\Omega$ if

$$
\varphi(\alpha \boldsymbol{x}+(1-\alpha) \boldsymbol{y}) \leq \alpha \varphi(\boldsymbol{x})+(1-\alpha) \varphi(\boldsymbol{y})
$$

for all $\boldsymbol{x}, \boldsymbol{y} \in \Omega$, and all $\alpha \in[0,1] . \varphi$ is said to be a concave function on $\Omega$ if and only if $-\varphi$ is convex function on $\Omega$.

Definition 4. [1, 2]

(i) A set $\Omega \subset \mathbb{R}^{n}$ is called a symmetric set, if $\boldsymbol{x} \in \Omega$ implies $\boldsymbol{x} P \in \Omega$ for every $n \times n$ permutation matrix $P$.

(ii) A function $\varphi: \Omega \rightarrow \mathbb{R}$ is called symmetric if for every permutation matrix $P, \varphi(\boldsymbol{x} P)=\varphi(\boldsymbol{x})$ for all $\boldsymbol{x} \in \Omega$. 
Lemma 1. (Schur-convex function decision theorem) [1, 2] Let $\Omega \subset \mathbb{R}^{n}$ be symmetric and have a nonempty interior convex set. $\Omega^{\circ}$ is the interior of $\Omega . \varphi: \Omega \rightarrow \mathbb{R}$ is continuous on $\Omega$ and differentiable in $\Omega^{\circ}$. Then $\varphi$ is the Schur - convex (or Schur - concave, respectively) function if and only if $\varphi$ is symmetric on $\Omega$ and

$$
\left(x_{1}-x_{2}\right)\left(\frac{\partial \varphi}{\partial x_{1}}-\frac{\partial \varphi}{\partial x_{2}}\right) \geq 0(\text { or } \leq 0, \text { respectively })
$$

holds for any $\boldsymbol{x} \in \Omega^{\circ}$.

The first systematical study of the functions preserving the ordering of majorization was made by Issai Schur in 1923. In Schur's honor, such functions are said to be "Schur-convex". It can be used extensively in analytic inequalities, combinatorial optimization, quantum physics, information theory, and other related fields. See [1].

Definition 5. 3] Let $\boldsymbol{x}=\left(x_{1}, x_{2}, \ldots, x_{n}\right) \in \mathbb{R}_{+}^{n}$ and $\boldsymbol{y}=\left(y_{1}, y_{2}, \ldots, y_{n}\right) \in \mathbb{R}_{+}^{n}$.

(i) A set $\Omega \subset \mathbb{R}_{+}^{n}$ is called a geometrically convex set if $\left(x_{1}^{\alpha} y_{1}^{\beta}, x_{2}^{\alpha} y_{2}^{\beta}, \ldots, x_{n}^{\alpha} y_{n}^{\beta}\right) \in \Omega$ for all $\boldsymbol{x}, \boldsymbol{y} \in \Omega$ and $\alpha, \beta \in[0,1]$ such that $\alpha+\beta=1$.

(ii) Let $\Omega \subset \mathbb{R}_{+}^{n}$. The function $\varphi: \Omega \rightarrow \mathbb{R}_{+}$is said to be Schur-geometrically convex function on $\Omega$ if $\left(\log x_{1}, \log x_{2}, \ldots, \log x_{n}\right) \prec\left(\log y_{1}, \log y_{2}, \ldots\right.$,

$\left.\log y_{n}\right)$ on $\Omega$ implies $\varphi(\boldsymbol{x}) \leq \varphi(\boldsymbol{y})$. The function $\varphi$ is said to be a Schur-geometrically concave function on $\Omega$ if and only if $-\varphi$ is Schur-geometrically convex function on $\Omega$.

We can obtain the following result immediately from Definitions 5.

Proposition 7. Let $\Omega \subset \mathbb{R}_{+}^{n}$ be a set, and let $\log \Omega=\left\{\left(\log x_{1}, \log x_{2}, \ldots, \log x_{n}\right):\left(x_{1}, x_{2}, \ldots, x_{n}\right) \in \Omega\right\}$. Then $\varphi: \Omega \rightarrow \mathbb{R}_{+}$is a Schur-geometrically convex (or Schur-geometrically concave, respectively) function on $\Omega$ if and only if $\varphi\left(e^{x_{1}}, e^{x_{2}}, \ldots, e^{x_{n}}\right)$ is a Schur-convex (or Schur-concave, respectively) function on $\log \Omega$.

Lemma 2. (Schur-geometrically convex function decision theorem) [3] Let $\Omega \subset \mathbb{R}_{+}^{n}$ be a symmetric and geometrically convex set with a nonempty interior $\Omega^{\circ}$. Let $\varphi: \Omega \rightarrow \mathbb{R}_{+}$be continuous on $\Omega$ and differentiable in $\Omega^{\circ}$. If $\varphi$ is symmetric on $\Omega$ and

$$
\left(\log x_{1}-\log x_{2}\right)\left(x_{1} \frac{\partial \varphi}{\partial x_{1}}-x_{2} \frac{\partial \varphi}{\partial x_{2}}\right) \geq 0 \quad(\text { or } \leq 0, \text { respectively })
$$

holds for any $\boldsymbol{x}=\left(x_{1}, x_{2}, \ldots, x_{n}\right) \in \Omega^{\circ}$, then $\varphi$ is a Schur-geometrically convex ( or Schur-geometrically concave, respectively) function.

The Schur-geometric convexity was proposed by Zhang [3] in 2004, and was investigated by Chu et al. [4], Guan [5], Sun et al. [6], and so on. We also note that some authors use the term "Schur multiplicative convexity".

In 2009, Chu ([7], [8], 9]) introduced the notion of Schur-harmonically convex function, and some interesting inequalities were obtained.

Definition 6. [7] Let $\Omega \subset \mathbb{R}_{+}^{n}$ or $\Omega \subset \mathbb{R}_{-}^{n}$.

(i) A set $\Omega$ is said to be harmonically convex if $\frac{\boldsymbol{x} \boldsymbol{y}}{\lambda \boldsymbol{x}+(1-\lambda) \boldsymbol{y}} \in \Omega$ for every $\boldsymbol{x}, \boldsymbol{y} \in \Omega$ and $\lambda \in[0,1]$, where $\boldsymbol{x} \boldsymbol{y}=\sum_{i=1}^{n} x_{i} y_{i}$ and $\frac{1}{\boldsymbol{x}}=\left(\frac{1}{x_{1}}, \frac{1}{x_{2}}, \ldots, \frac{1}{x_{n}}\right)$. 
(ii) A function $\varphi: \Omega \rightarrow \mathbb{R}_{+}$is said to be Schur-harmonically convex on $\Omega$ if $\frac{1}{\boldsymbol{x}} \prec \frac{1}{\boldsymbol{y}}$ implies $\varphi(\boldsymbol{x}) \leq \varphi(\boldsymbol{y})$. A function $\varphi$ is said to be a Schur-harmonically concave function on $\Omega$ if and only if $-\varphi$ is a Schurharmonically convex function.

By Definitions 6, the following is obvious.

Proposition 8. Let $\Omega \subset \mathbb{R}_{+}^{n}$ be a set, and let $\frac{1}{\Omega}=\left\{\left(\frac{1}{x_{1}}, \frac{1}{x_{2}}, \ldots, \frac{1}{x_{n}}\right)\right.$ :

$\left.\left(x_{1}, x_{2}, \ldots, x_{n}\right) \in \Omega\right\}$. Then $\varphi: \Omega \rightarrow \mathbb{R}_{+}$is a Schur-harmonically convex (or Schur-harmonically concave, respectively) function on $\Omega$ if and only if $\varphi\left(\frac{1}{x_{1}}, \frac{1}{x_{2}}, \ldots, \frac{1}{x_{n}}\right)$ is a Schur-convex (or Schur-concave, respectively) function on $\frac{1}{\Omega}$.

Lemma 3. ( Schur-harmonically convex function decision theorem) [7] Let $\Omega \subset \mathbb{R}_{+}^{n}$ or $\Omega \subset \mathbb{R}_{-}^{n}$ be a symmetric and harmonically convex set with inner points and let $\varphi: \Omega \rightarrow \mathbb{R}$ be a continuously symmetric function which is differentiable on $\Omega^{\circ}$. Then $\varphi$ is Schur-harmonically convex (or Schur-harmonically concave, respectively) on $\Omega$ if and only if

$$
\left(x_{1}-x_{2}\right)\left(x_{1}^{2} \frac{\partial \varphi(\boldsymbol{x})}{\partial x_{1}}-x_{2}^{2} \frac{\partial \varphi(\boldsymbol{x})}{\partial x_{2}}\right) \geq 0 \quad(\text { or } \leq 0, \text { respectively }), \quad \boldsymbol{x} \in \Omega^{\circ} .
$$

Remark 1. We extend the definition and determination theorem of Schur-harmonically convex function established by Chu as follows:

(i) The set $\Omega \subset \mathbb{R}_{+}^{n}$ is extended to $\Omega \subset \mathbb{R}_{+}^{n}$ or $\Omega \subset \mathbb{R}_{-}^{n}$;

(ii) The function $\varphi: \Omega \rightarrow \mathbb{R}$ must not be a positive function.

Lemma 4. ([1], [2]) Let the set $\mathcal{A}, \mathcal{B} \subset \mathbb{R}, \varphi: \mathcal{B}^{n} \rightarrow \mathbb{R}, f: \mathcal{A} \rightarrow \mathcal{B}$ and $\psi\left(x_{1}, x_{2}, \ldots, x_{n}\right)=\varphi\left(f\left(x_{1}\right), f\left(x_{2}\right), \ldots, f\left(x_{n}\right)\right)$ $\mathcal{A}^{n} \rightarrow \mathbb{R}$

(i) If $f$ is convex and $\varphi$ is increasing and Schur-convex, then $\psi$ is Schur-convex;

(ii) If $f$ is convex and $\varphi$ is decreasing and Schur-concave, then $\psi$ is Schur-concave.

Lemma 5. [3, [26] Let the set $\Omega \subset \mathbb{R}_{+}^{n}$. The function $\varphi: \Omega \rightarrow \mathbb{R}_{+}$is differentiable.

(i) If $\varphi$ is increasing and Schur-convex or Schur-geometrically convex, then $\varphi$ is Schur-harmonically convex.

(ii) If $\varphi$ is decreasing and Schur-geometrically concave, then $\varphi$ is Schur-harmonically concave.

Lemma 6. [1] Let $\boldsymbol{x}=\left(x_{1}, x_{2}, \ldots, x_{n}\right) \in \mathbb{R}_{+}^{n}, n \geq 2,0<r \leq s$. Then

$$
\left(\frac{x_{1}^{r}}{\sum_{j=1}^{n} x_{j}^{r}}, \frac{x_{2}^{r}}{\sum_{j=1}^{n} x_{j}^{r}}, \ldots, \frac{x_{n}^{r}}{\sum_{j=1}^{n} x_{j}^{r}}\right) \prec\left(\frac{x_{1}^{s}}{\sum_{j=1}^{n} x_{j}^{s}}, \frac{x_{2}^{s}}{\sum_{j=1}^{n} x_{j}^{s}}, \ldots, \frac{x_{n}^{s}}{\sum_{j=1}^{n} x_{j}^{s}}\right) .
$$

Lemma 7. [1] Let $\boldsymbol{x}=\left(x_{1}, x_{2}, \ldots, x_{n}\right) \in \mathbb{R}_{+}^{n}, n \geq 2, \sum_{i=1}^{n} x_{i}=s>0, c \geq s$. Then

$$
\left(\frac{c-x_{1}}{n c-s}, \frac{c-x_{2}}{n c-s}, \ldots, \frac{c-x_{n}}{n c-s}\right) \prec\left(\frac{x_{1}}{s}, \frac{x_{2}}{s}, \ldots, \frac{x_{n}}{s}\right) .
$$




\section{Proofs of main results}

\section{Proof of Theorem 1:}

for $r=1$ and $r=2$, it is easy to prove that $c_{n}^{*}\left(\frac{\boldsymbol{x}}{\boldsymbol{x}-1}, r\right)$ is Schur-convex on $(1,+\infty)^{n}$.

Now consider the case of $r \geq 3$. By the symmetry of $c_{n}^{*}\left(\frac{x}{\boldsymbol{x}-1}, r\right)$, without loss of generality, we can set $x_{1}>x_{2}$.

$$
\begin{aligned}
c_{n}^{*}\left(\frac{\boldsymbol{x}}{\boldsymbol{x}-1}, r\right) & =\prod_{\substack{i_{1}+i_{2}+\cdots+i_{n}=r \\
i_{1} \neq 0, i_{2}=0}} \sum_{j=1}^{n} \frac{i_{j} x_{j}}{x_{j}-1} \times \prod_{\substack{i_{1}+i_{2}+\cdots+i_{n}=r \\
i_{1}=0, i_{2} \neq 0}} \sum_{j=1}^{n} \frac{i_{j} x_{j}}{x_{j}-1} \\
& \times \prod_{\substack{i_{1}+i_{2}+\cdots+i_{n}=r \\
i_{1} \neq 0, i_{2} \neq 0}} \sum_{j=1}^{n} \frac{i_{j} x_{j}}{x_{j}-1} \times \prod_{\substack{i_{1}+i_{2}+\cdots,+i_{n}=r \\
i_{1}=0, i_{2}=0}} \sum_{j=1}^{n} \frac{i_{j} x_{j}}{x_{j}-1} .
\end{aligned}
$$

Then

$$
\begin{aligned}
& \frac{\partial c_{n}^{*}\left(\frac{\boldsymbol{x}}{\boldsymbol{x}-1}, r\right)}{\partial x_{1}}=c_{n}^{*}\left(\frac{\boldsymbol{x}}{\boldsymbol{x}-1}, r\right) \\
& \times\left(\sum_{\substack{i_{1}+i_{2}+\cdots+i_{n}=r \\
i_{1} \neq 0, i_{2}=0}} \frac{-i_{1}}{\left(x_{1}-1\right)^{2} \sum_{j=1}^{n} \frac{i_{j} x_{j}}{x_{j}-1}}+\sum_{\substack{i_{1}+i_{2}+\cdots+i_{n}=r \\
i_{1} \neq 0, i_{2} \neq 0}} \frac{-i_{1}}{\left(x_{1}-1\right)^{2} \sum_{j=1}^{n} \frac{i_{j} x_{j}}{x_{j}-1}}\right) \\
& =c_{n}^{*}\left(\frac{\boldsymbol{x}}{\boldsymbol{x}-1}, r\right)\left(\sum_{\substack{k+k_{3}+\ldots+k_{n}=r \\
k \neq 0}} \frac{-k}{\left(x_{1}-1\right)^{2}\left(\frac{k x_{1}}{x_{1}-1}+\sum_{j=3}^{n} \frac{k_{j} x_{j}}{x_{j}-1}\right)}\right. \\
& \left.+\sum_{\substack{k+m+i_{3}+\cdots+i_{n}=r \\
k \neq 0, m \neq 0}} \frac{-k}{\left(x_{1}-1\right)^{2}\left(\frac{k x_{1}}{x_{1}-1}+\frac{m x_{2}}{x_{2}-1}+\sum_{j=3}^{n} \frac{k_{j} x_{j}}{x_{j}-1}\right)}\right) .
\end{aligned}
$$

By the same arguments,

$$
\begin{aligned}
\frac{\partial c_{n}^{*}\left(\frac{\boldsymbol{x}}{\boldsymbol{x}-1}, r\right)}{\partial x_{2}} & =c_{n}^{*}\left(\frac{\boldsymbol{x}}{\boldsymbol{x}-1}, r\right)\left(\sum_{\substack{k+k_{3}+\cdots+k_{n}=r \\
k \neq 0}} \frac{-k}{\left(x_{2}-1\right)^{2}\left(\frac{k x_{2}}{x_{2}-1}+\sum_{j=3}^{n} \frac{k_{j} x_{j}}{x_{j}-1}\right)}\right. \\
& +\sum_{\substack{k+m+i_{3}+\cdots+i_{n}=r \\
k \neq 0, m \neq 0}} \frac{-k}{\left(x_{2}-1\right)^{2}\left(\frac{k x_{2}}{x_{2}-1}+\frac{m x_{1}}{x_{1}-1}+\sum_{j=3}^{n} \frac{k_{j} x_{j}}{x_{j}-1}\right)},
\end{aligned}
$$

then

$$
\frac{\partial c_{n}^{*}\left(\frac{\boldsymbol{x}}{\boldsymbol{x}-1}, r\right)}{\partial x_{1}}-\frac{\partial c_{n}^{*}\left(\frac{\boldsymbol{x}}{\boldsymbol{x}-1}, r\right)}{\partial x_{2}}=c_{n}^{*}\left(\frac{\boldsymbol{x}}{\boldsymbol{x}-1}, r\right)\left(A_{1}+A_{2}\right),
$$


where

$$
\begin{aligned}
A_{1} & =\sum_{\substack{k+k_{3}+\ldots+k_{n}=r \\
k \neq 0}}\left(\frac{-k}{\left(x_{1}-1\right)^{2}\left(\frac{k x_{1}}{x_{1}-1}+\sum_{j=3}^{n} \frac{k_{j} x_{j}}{x_{j}-1}\right)}-\frac{-k}{\left(x_{2}-1\right)^{2}\left(\frac{k x_{2}}{x_{2}-1}+\sum_{j=3}^{n} \frac{k_{j} x_{j}}{x_{j}-1}\right)}\right) \\
& =k \sum_{\substack{k+k_{3}+\cdots+k_{n}=r \\
k \neq 0}} \frac{k\left(x_{1}+x_{2}-1\right)\left(x_{1}-x_{2}\right)+\left(x_{1}-x_{2}\right)\left(2-x_{1}-x_{2}\right) \sum_{j=3}^{n} \frac{k_{j} x_{j}}{x_{j}-1}}{\left(x_{1}-1\right)^{2}\left(\frac{k x_{1}}{x_{1}-1}+\sum_{j=3}^{n} \frac{k_{j} x_{j}}{x_{j}-1}\right)\left(x_{2}-1\right)^{2}\left(\frac{k x_{2}}{x_{2}-1}+\sum_{j=3}^{n} \frac{k_{j} x_{j}}{x_{j}-1}\right)}
\end{aligned}
$$

and

$$
\begin{aligned}
A_{2}= & \sum_{\substack{k+m+i_{3}+\cdots+i_{n}=r \\
k \neq 0, m \neq 0}}\left(\frac{-k}{\left(x_{1}-1\right)^{2}\left(\frac{k x_{1}}{x_{1}-1}+\frac{m x_{2}}{x_{2}-1}+\sum_{j=3}^{n} \frac{k_{j} x_{j}}{x_{j}-1}\right)}-\frac{-k}{\left(x_{2}-1\right)^{2}\left(\frac{k x_{2}}{x_{2}-1}+\frac{m x_{1}}{x_{1}-1}+\sum_{j=3}^{n} \frac{k_{j} x_{j}}{x_{j}-1}\right)}\right) \\
& =k \sum_{\substack{k+m+i_{3}+\cdots+i_{n}=r \\
k \neq 0, m \neq 0}} \frac{\lambda_{1}}{\left(x_{1}-1\right)^{2}\left(\frac{k x_{1}}{x_{1}-1}+\frac{m x_{2}}{x_{2}-1}+\sum_{j=3}^{n} \frac{k_{j} x_{j}}{x_{j}-1}\right)\left(x_{2}-1\right)^{2}\left(\frac{k x_{2}}{x_{2}-1}+\frac{m x_{1}}{x_{1}-1}+\sum_{j=3}^{n} \frac{k_{j} x_{j}}{x_{j}-1}\right)},
\end{aligned}
$$

where

$$
\lambda_{1}=k\left(x_{1}+x_{2}-1\right)\left(x_{1}-x_{2}\right)+\left(\frac{\left(1-x_{2}\right)^{2} m x_{1}}{1-x_{1}}-\frac{\left(1-x_{1}\right)^{2} m x_{2}}{1-x_{2}}\right)+\left(x_{1}-x_{2}\right)\left(x_{1}+x_{2}-2\right) \sum_{j=3}^{n} \frac{k_{j} x_{j}}{x_{j}-1} .
$$

Let $f(t)=\frac{(1-t)^{3}}{m t}$. Then $f^{\prime}(t)=-\frac{m(1+2 t)(1-t)^{2}}{m^{2} t^{2}} \leq 0$, this means that $f(t)$ is descending on $\mathbb{R}_{+}$. So that $\frac{\left(1-x_{1}\right)^{3}}{m x_{1}} \leq \frac{\left(1-x_{2}\right)^{3}}{m x_{2}}$, namely $\frac{\left(1-x_{2}\right)^{2} m x_{1}}{1-x_{1}}-\frac{\left.m^{2} t^{2}-x_{1}\right)^{2} m x_{2}}{1-x_{2}} \geq 0$. It is easy to see that $A_{1} \geq 0$ and $A_{2} \geq 0$ for $\boldsymbol{x} \in(1,+\infty)^{n}$, so

$$
\frac{\partial c_{n}^{*}\left(\frac{\boldsymbol{x}}{\boldsymbol{x}-1}, r\right)}{\partial x_{1}}-\frac{\partial c_{n}^{*}\left(\frac{\boldsymbol{x}}{\boldsymbol{x}-1}, r\right)}{\partial x_{2}} \geq 0
$$

by Lemma 1 , it follows that $c_{n}^{*}\left(\frac{\boldsymbol{x}}{1-\boldsymbol{x}}, r\right)$ is Schur-convex on $(1,+\infty)^{n}$.

From (11) and (12), it follows that

$$
x_{1} \frac{\partial c_{n}^{*}\left(\frac{\boldsymbol{x}}{\boldsymbol{x}-1}, r\right)}{\partial x_{1}}-x_{2} \frac{\partial c_{n}^{*}\left(\frac{\boldsymbol{x}}{\boldsymbol{x}-1}, r\right)}{\partial x_{2}}=c_{n}^{*}\left(\frac{\boldsymbol{x}}{\boldsymbol{x}-1}, r\right)\left(B_{1}+B_{2}\right),
$$

where

$$
\begin{aligned}
B_{1} & =\sum_{\substack{k+k_{3}+\ldots+k_{n}=r \\
k \neq 0}}\left(\frac{-k x_{1}}{\left(x_{1}-1\right)^{2}\left(\frac{k x_{1}}{x_{1}-1}+\sum_{j=3}^{n} \frac{k_{j} x_{j}}{x_{j}-1}\right)}-\frac{-k x_{2}}{\left(x_{2}-1\right)^{2}\left(\frac{k x_{2}}{x_{2}-1}+\sum_{j=3}^{n} \frac{k_{j} x_{j}}{x_{j}-1}\right)}\right) \\
& =k \sum_{\substack{k+k_{3}+\cdots+k_{n}=r \\
k \neq 0}} \frac{k x_{1} x_{2}\left(x_{1}-x_{2}\right)+\left(x_{1}-x_{2}\right)\left(x_{1} x_{2}-1\right) \sum_{j=3}^{n} \frac{k_{j} x_{j}}{x_{j}-1}}{\left(x_{1}-1\right)^{2}\left(\frac{k x_{1}}{x_{1}-1}+\sum_{j=3}^{n} \frac{k_{j} x_{j}}{x_{j}-1}\right)\left(x_{2}-1\right)^{2}\left(\frac{k x_{2}}{x_{2}-1}+\sum_{j=3}^{n} \frac{k_{j} x_{j}}{x_{j}-1}\right)}
\end{aligned}
$$


and

$$
\begin{aligned}
B_{2}= & \sum_{\substack{k+m+i_{3}+\cdots+i_{n}=r \\
k \neq 0, m \neq 0}}\left(\frac{-k x_{1}}{\left(x_{1}-1\right)^{2}\left(\frac{k x_{1}}{x_{1}-1}+\frac{m x_{2}}{x_{2}-1}+\sum_{j=3}^{n} \frac{k_{j} x_{j}}{x_{j}-1}\right)}-\frac{-k x_{2}}{\left(x_{2}-1\right)^{2}\left(\frac{k x_{2}}{x_{2}-1}+\frac{m x_{1}}{x_{1}-1}+\sum_{j=3}^{n} \frac{k_{j} x_{j}}{x_{j}-1}\right)}\right) \\
& =k \sum_{\substack{k+m+i_{3}+\cdots+i_{n}=r \\
k \neq 0, m \neq 0}} \frac{\lambda_{2}}{\left(x_{1}-1\right)^{2}\left(\frac{k x_{1}}{x_{1}-1}+\frac{m x_{2}}{x_{2}-1}+\sum_{j=3}^{n} \frac{k_{j} x_{j}}{x_{j}-1}\right)\left(x_{2}-1\right)^{2}\left(\frac{k x_{2}}{x_{2}-1}+\frac{m x_{1}}{x_{1}-1}+\sum_{j=3}^{n} \frac{k_{j} x_{j}}{x_{j}-1}\right)},
\end{aligned}
$$

where

$$
\lambda_{2}=k x_{1} x_{2}\left(x_{1}-x_{2}\right)+\left(\frac{\left(x_{1}-1\right)^{2} m x_{2}^{2}}{x_{2}-1}-\frac{\left(x_{2}-1\right)^{2} m x_{1}^{2}}{x_{1}-1}\right)+\left(x_{1}-x_{2}\right)\left(x_{1} x_{2}-1\right) \sum_{j=3}^{n} \frac{k_{j} x_{j}}{x_{j}-1} .
$$

Let $g(t)=\frac{(t-1)^{3}}{m t^{2}}$. Then $g^{\prime}(t)=\frac{m t(t+2)(t-1)^{2}}{m^{2} t^{4}} \geq 0$, this means that $g(t)$ is increasing on $\mathbb{R}_{+}$. So that $\frac{\left(x_{1}-1\right)^{3}}{m x_{1}^{2}} \geq \frac{\left(x_{2}-1\right)^{3}}{m x_{2}^{2}}$, namely $\frac{\left(x_{1}-1\right)^{2} m x_{2}^{2}}{x_{2}-1}-\frac{\left(x_{2}-1\right)^{2} m x_{1}^{2}}{x_{1}-1} \geq 0$. It is easy to see that $B_{1} \geq 0$ and $B_{2} \geq 0$ for $\boldsymbol{x} \in(1,+\infty)^{n}$, so

$$
x_{1} \frac{\partial c_{n}^{*}\left(\frac{\boldsymbol{x}}{\boldsymbol{x}-1}, r\right)}{\partial x_{1}}-x_{2} \frac{\partial c_{n}^{*}\left(\frac{\boldsymbol{x}}{\boldsymbol{x}-1}, r\right)}{\partial x_{2}} \geq 0,
$$

by Lemma 2 , it follows that $c_{n}^{*}\left(\frac{\boldsymbol{x}}{\boldsymbol{x}-1}, r\right)$ is Schur-geometrically convex on $(1,+\infty)^{n}$.

From (11) and (12), it follows that

$$
x_{1}^{2} \frac{\partial c_{n}^{*}\left(\frac{\boldsymbol{x}}{\boldsymbol{x}-1}, r\right)}{\partial x_{1}}-x_{2}^{2} \frac{\partial c_{n}^{*}\left(\frac{\boldsymbol{x}}{\boldsymbol{x}-1}, r\right)}{\partial x_{2}}=c_{n}^{*}\left(\frac{\boldsymbol{x}}{\boldsymbol{x}-1}, r\right)\left(C_{1}+C_{2}\right),
$$

where

$$
\begin{aligned}
C_{1} & =\sum_{\substack{k+k_{3}+\ldots+k_{n}=r \\
k \neq 0}}\left(\frac{-k x_{1}^{2}}{\left(x_{1}-1\right)^{2}\left(\frac{k x_{1}}{x_{1}-1}+\sum_{j=3}^{n} \frac{k_{j} x_{j}}{x_{j}-1}\right)}-\frac{-k x_{2}^{2}}{\left(x_{2}-1\right)^{2}\left(\frac{k x_{2}}{x_{2}-1}+\sum_{j=3}^{n} \frac{k_{j} x_{j}}{x_{j}-1}\right)}\right) \\
& =k \sum_{\substack{k+k_{3}+\cdots+k_{n}=r \\
k \neq 0}} \frac{k x_{1} x_{2}\left(x_{1}-x_{2}\right)+\left(x_{1}-x_{2}\right)\left(2 x_{1} x_{2}-x_{1}-x_{2}\right) \sum_{j=3}^{n} \frac{k_{j} x_{j}}{x_{j}-1}}{\left(x_{1}-1\right)^{2}\left(\frac{k x_{1}}{x_{1}}+\sum_{j=3}^{n} \frac{k_{j} x_{j}}{x_{j}-1}\right)\left(x_{2}-1\right)^{2}\left(\frac{k x_{2}}{x_{2}-1}+\sum_{j=3}^{n} \frac{k_{j} x_{j}}{x_{j}-1}\right)}
\end{aligned}
$$

and

$$
\begin{aligned}
C_{2}= & \sum_{\substack{k+m+i_{3}+\cdots+i_{n}=r \\
k \neq 0, m \neq 0}}\left(\frac{-k x_{1}^{2}}{\left(x_{1}-1\right)^{2}\left(\frac{k x_{1}}{x_{1}-1}+\frac{m x_{2}}{x_{2}-1}+\sum_{j=3}^{n} \frac{k_{j} x_{j}}{x_{j}-1}\right)}-\frac{-k x_{2}^{2}}{\left(x_{2}-1\right)^{2}\left(\frac{k x_{2}}{x_{2}-1}+\frac{m x_{1}}{x_{1}-1}+\sum_{j=3}^{n} \frac{k_{j} x_{j}}{x_{j}-1}\right)}\right) \\
& =k \sum_{\substack{k+m+i_{3}+\cdots+i_{n}=r \\
k \neq 0, m \neq 0}} \frac{\lambda_{3}}{\left(x_{1}-1\right)^{2}\left(\frac{k x_{1}}{x_{1}-1}+\frac{m x_{2}}{x_{2}-1}+\sum_{j=3}^{n} \frac{k_{j} x_{j}}{x_{j}-1}\right)\left(x_{2}-1\right)^{2}\left(\frac{k x_{2}}{x_{2}-1}+\frac{m x_{1}}{x_{1}-1}+\sum_{j=3}^{n} \frac{k_{j} x_{j}}{x_{j}-1}\right)},
\end{aligned}
$$

where

$$
\lambda_{3}=k x_{1} x_{2}\left(x_{1}-x_{2}\right)+\left(\frac{\left(x_{1}-1\right)^{2} m x_{2}^{3}}{x_{2}-1}-\frac{\left(x_{2}-1\right)^{2} m x_{1}^{3}}{x_{1}-1}\right)+\left(x_{1}-x_{2}\right)\left(2 x_{1} x_{2}-x_{1}-x_{2}\right) \sum_{j=3}^{n} \frac{k_{j} x_{j}}{x_{j}-1} .
$$


Let $h(t)=\frac{(t-1)^{3}}{m t^{3}}$. Then $h^{\prime}(t)=\frac{3 m t^{2}(t-1)^{2}}{m^{2} t^{6}} \geq 0$, this means that $h(t)$ is increasing on $\mathbb{R}$. So that $\frac{\left(x_{1}-1\right)^{3}}{m x_{1}^{3}} \geq \frac{\left(x_{2}-1\right)^{3}}{m x_{2}^{3}}$, namely $\frac{\left(x_{1}-1\right)^{2} m x_{2}^{3}}{x_{2}-1}-\frac{\left(x_{2}-1\right)^{2} m x_{1}^{3}}{x_{1}-1} \geq 0$. It is easy to see that $C_{1} \geq 0$ and $C_{2} \geq 0$ for $\boldsymbol{x} \in(1,+\infty)^{n}$, so

$$
x_{1}^{2} \frac{\partial c_{n}^{*}\left(\frac{\boldsymbol{x}}{\boldsymbol{x}-1}, r\right)}{\partial x_{1}}-x_{2}^{2} \frac{\partial c_{n}^{*}\left(\frac{\boldsymbol{x}}{\boldsymbol{x}-1}, r\right)}{\partial x_{2}} \geq 0,
$$

by Lemma 3, it follows that $c_{n}^{*}\left(\frac{\boldsymbol{x}}{\boldsymbol{x}-1}, r\right)$ is Schur-harmonically convex on $(1,+\infty)^{n}$.

The proof of Theorem 1 is completed.

\section{Proof of Theorem 2:}

(i) Let $p(t)=\frac{t}{1-t}$. Then

$$
p^{\prime}(t)=\frac{1}{(1-t)^{2}}, \quad p^{\prime \prime}(t)=\frac{2}{(1-t)^{3}} .
$$

From Proposition 4 , we know that $c_{n}^{*}(\boldsymbol{x}, r)$ is increasing on $\mathbb{R}_{+}^{n}$, but $p(t)$ is increasing on $\mathbb{R}$, therefore, the function $c_{n}^{*}\left(\frac{\boldsymbol{x}}{1-\boldsymbol{x}}, r\right)$ is increasing on $\mathbb{R}_{+}^{n}$.

For the case of $r=1$ and $r=2$, it is easy to prove that $c_{n}^{*}\left(\frac{x}{1-x}, r\right)$ is Schur-convex on $\left[\frac{1}{2}, 1\right)^{n}$.

Now consider the case of $r \geq 3$. By the symmetry of $c_{n}^{*}\left(\frac{x}{1-x}, r\right)$, without loss of generality, we can set $x_{1}>x_{2}$.

$$
\begin{aligned}
c_{n}^{*}\left(\frac{\boldsymbol{x}}{1-\boldsymbol{x}}, r\right) & =\prod_{\substack{i_{1}+i_{2}+\cdots+i_{n}=r \\
i_{1} \neq 0, i_{2}=0}} \sum_{j=1}^{n} \frac{i_{j} x_{j}}{1-x_{j}} \times \prod_{\substack{i_{1}+i_{2}+\cdots+i_{n}=r \\
i_{1}=0, i_{2} \neq 0}} \sum_{j=1}^{n} \frac{i_{j} x_{j}}{1-x_{j}} \\
& \times \prod_{\substack{i_{1}+i_{2}+\cdots+i_{n}=r \\
i_{1} \neq 0, i_{2} \neq 0}} \sum_{j=1}^{n} \frac{i_{j} x_{j}}{1-x_{j}} \times \prod_{\substack{i_{1}+i_{2}+\cdots+i_{n}=r \\
i_{1}=0, i_{2}=0}} \sum_{j=1}^{n} \frac{i_{j} x_{j}}{1-x_{j}} .
\end{aligned}
$$

Then

$$
\begin{aligned}
& \frac{\partial c_{n}^{*}\left(\frac{\boldsymbol{x}}{1-\boldsymbol{x}}, r\right)}{\partial x_{1}}=c_{n}^{*}\left(\frac{\boldsymbol{x}}{1-\boldsymbol{x}}, r\right) \\
& \times\left(\sum_{\substack{i_{1}+i_{2}+\cdots+i_{n}=r \\
i_{1} \neq 0, i_{2}=0}} \frac{i_{1}}{\left(1-x_{1}\right)^{2} \sum_{j=1}^{n} \frac{i_{j} x_{j}}{1-x_{j}}}+\sum_{\substack{i_{1}+i_{2}+\cdots+i_{n}=r \\
i_{1} \neq 0, i_{2} \neq 0}} \frac{i_{1}}{\left(1-x_{1}\right)^{2} \sum_{j=1}^{n} \frac{i_{j} x_{j}}{1-x_{j}}}\right) \\
& =c_{n}^{*}\left(\frac{\boldsymbol{x}}{1-\boldsymbol{x}}, r\right) \\
& +\sum_{\substack{k+k_{3}+\cdots+k_{n}=r \\
k \neq 0}}^{\left(\sum_{\substack{k+m+i_{3}+\cdots+i_{n}=r \\
k \neq 0, m \neq 0}} \frac{k}{\left(1-x_{1}\right)^{2}\left(\frac{k x_{1}}{1-x_{1}}+\sum_{j=3}^{n} \frac{k_{j} x_{j}}{1-x_{j}}\right)}\right.}
\end{aligned}
$$


By the same arguments,

$$
\begin{gathered}
\frac{\partial c_{n}^{*}\left(\frac{\boldsymbol{x}}{1-\boldsymbol{x}}, r\right)}{\partial x_{2}}=c_{n}^{*}\left(\frac{\boldsymbol{x}}{1-\boldsymbol{x}}, r\right)\left(\sum_{\substack{k+k_{3}+\cdots+k_{n}=r \\
k \neq 0}} \frac{k}{\left(1-x_{2}\right)^{2}\left(\frac{k x_{2}}{1-x_{2}}+\sum_{j=3}^{n} \frac{k_{j} x_{j}}{1-x_{j}}\right)}\right. \\
\left.+\sum_{\substack{k+m+i_{3}+\cdots+i_{n}=r \\
k \neq 0, m \neq 0}} \frac{k}{\left(1-x_{2}\right)^{2}\left(\frac{k x_{2}}{1-x_{2}}+\frac{m x_{1}}{1-x_{1}}+\sum_{j=3}^{n} \frac{k_{j} x_{j}}{1-x_{j}}\right)}\right), \\
\frac{\partial c_{n}^{*}\left(\frac{\boldsymbol{x}}{1-\boldsymbol{x}}, r\right)}{\partial x_{1}}-\frac{\partial c_{n}^{*}\left(\frac{\boldsymbol{x}}{1-\boldsymbol{x}}, r\right)}{\partial x_{2}}=c_{n}^{*}\left(\frac{\boldsymbol{x}}{1-\boldsymbol{x}}, r\right)\left(D_{1}+D_{2}\right),
\end{gathered}
$$

where

$$
\begin{aligned}
D_{1} & =\sum_{\substack{k+k_{3}+\cdots+k_{n}=r \\
k \neq 0}}\left(\frac{k}{\left(1-x_{1}\right)^{2}\left(\frac{k}{1-x_{1}}+\sum_{j=3}^{n} \frac{k_{j} x_{j}}{1-x_{j}}\right)}-\frac{k}{\left(1-x_{2}\right)^{2}\left(\frac{k x_{2}}{1-x_{2}}+\sum_{j=3}^{n} \frac{k_{j} x_{j}}{1-x_{j}}\right)}\right) \\
& =k \sum_{\substack{k+k_{3}+\ldots+k_{n}=r \\
k \neq 0}} \frac{k\left(x_{1}+x_{2}-1\right)\left(x_{1}-x_{2}\right)+\left(x_{1}-x_{2}\right)\left(2-x_{1}-x_{2}\right) \sum_{j=3}^{n} \frac{k_{j} x_{j}}{1-x_{j}}}{\left(1-x_{1}\right)^{2}\left(\frac{k x_{1}}{1-x_{1}}+\sum_{j=3}^{n} \frac{k_{j} x_{j}}{1-x_{j}}\right)\left(1-x_{2}\right)^{2}\left(\frac{k x_{2}}{1-x_{2}}+\sum_{j=3}^{n} \frac{k_{j} x_{j}}{1-x_{j}}\right)}
\end{aligned}
$$

and

$$
\begin{aligned}
D_{2}= & \sum_{\substack{k+m+i_{3}+\cdots+i_{n}=r \\
k \neq 0, m \neq 0}}\left(\frac{k}{\left(1-x_{1}\right)^{2}\left(\frac{k x_{1}}{1-x_{1}}+\frac{m x_{2}}{1-x_{2}}+\sum_{j=3}^{n} \frac{k_{j} x_{j}}{1-x_{j}}\right)}-\frac{k}{\left(1-x_{2}\right)^{2}\left(\frac{k x_{2}}{1-x_{2}}+\frac{m x_{1}}{1-x_{1}}+\sum_{j=3}^{n} \frac{k_{j} x_{j}}{1-x_{j}}\right)}\right) \\
& =k \sum_{\substack{k+m+i_{3}+\cdots+i_{n}=r \\
k \neq 0, m \neq 0}} \frac{\delta_{1}}{\left(1-x_{1}\right)^{2}\left(\frac{k x_{1}}{1-x_{1}}+\frac{m x_{2}}{1-x_{2}}+\sum_{j=3}^{n} \frac{k_{j} x_{j}}{1-x_{j}}\right)\left(1-x_{2}\right)^{2}\left(\frac{k x_{2}}{1-x_{2}}+\frac{m x_{1}}{1-x_{1}}+\sum_{j=3}^{n} \frac{k_{j} x_{j}}{1-x_{j}}\right)}
\end{aligned}
$$

where

$$
\delta_{1}=k\left(x_{1}+x_{2}-1\right)\left(x_{1}-x_{2}\right)+\left(\frac{\left(1-x_{2}\right)^{2} m x_{1}}{1-x_{1}}-\frac{\left(1-x_{1}\right)^{2} m x_{2}}{1-x_{2}}\right)+\left(x_{1}-x_{2}\right)\left(2-x_{1}-x_{2}\right) \sum_{j=3}^{n} \frac{k_{j} x_{j}}{1-x_{j}} .
$$

Let $q(t)=\frac{(1-t)^{3}}{m t}$. Then $q^{\prime}(t)=-\frac{m(1+2 t)(1-t)^{2}}{m^{2} t^{2}} \leq 0$, this means that $q(t)$ is descending on $\mathbb{R}_{+}$. So that $\frac{\left(1-x_{1}\right)^{3}}{m x_{1}} \leq \frac{\left(1-x_{2}\right)^{3}}{m x_{2}}$, namely $\frac{\left(1-x_{2}\right)^{2} m x_{1}}{1-x_{1}}-\frac{\left(1-x_{1}\right)^{2} m x_{2}}{1-x_{2}} \geq 0$. It is easy to see that $D_{1} \geq 0$ and $D_{2} \geq 0$ for $\boldsymbol{x} \in\left[\frac{1}{2}, 1\right)^{n}$, so

$$
\frac{\partial c_{n}^{*}\left(\frac{\boldsymbol{x}}{1-\boldsymbol{x}}, r\right)}{\partial x_{1}}-\frac{\partial c_{n}^{*}\left(\frac{\boldsymbol{x}}{1-\boldsymbol{x}}, r\right)}{\partial x_{2}} \geq 0
$$

by Lemma 1 , it follows that $c_{n}^{*}\left(\frac{\boldsymbol{x}}{1-\boldsymbol{x}}, r\right)$ is Schur-convex on $\left[\frac{1}{2}, 1\right)^{n}$.

(ii)

Notice that

$$
c_{n}^{*}\left(\frac{\boldsymbol{x}}{\boldsymbol{x}-1}, r\right)=(-1)^{r} c_{n}^{*}\left(\frac{\boldsymbol{x}}{1-\boldsymbol{x}}, r\right),
$$


combining with the Schur-convexity of $c_{n}^{*}\left(\frac{\boldsymbol{x}}{\boldsymbol{x}-1}, r\right)$ on $(1,+\infty)^{n}$ (see Theorem 1), we can prove $(i i)$ in Theorem 2.

(iii) For $t<0$, from (13), we have $p(t)<0, p^{\prime}(t)>0$ and $p^{\prime \prime}(t)>0$, this means that $p(t)$ is an increasing convex function with a negative value for $t<0$.

By Proposition 6, we know that if $r$ is an even integer, then $c_{n}^{*}(\boldsymbol{x}, r)$ is decreasing and Schur-concave on $\mathbb{R}_{-}^{n}$, from Lemma $5(i i)$, it follows that $c_{n}^{*}\left(\frac{\boldsymbol{x}}{1-\boldsymbol{x}}, r\right)$ is decreasing and Schur-concave on $\mathbb{R}_{-}^{n}$.

From Proposition 6, we know that if $r$ is an odd integer, then $c_{n}^{*}(\boldsymbol{x}, r)$ is increasing and Schur-convex on $\mathbb{R}_{-}^{n}$, by Lemma $5(i)$, it follows that $c_{n}^{*}\left(\frac{\boldsymbol{x}}{1-\boldsymbol{x}}, r\right)$ is increasing and Schur-convex on $\mathbb{R}_{-}^{n}$.

The proof of Theorem 2 is completed.

\section{Proof of Theorem 3:}

For $r=1$ and $r=2$, it is easy to prove that $c_{n}^{*}\left(\frac{\boldsymbol{x}}{1-\boldsymbol{x}}, r\right)$ is Schur-geometrically convex on $(0,1)^{n}$.

Now consider the case of $r \geq 3$. By the symmetry of $c_{n}^{*}\left(\frac{x}{1-x}, r\right)$, without loss of generality, we can set $x_{1}>x_{2}$.

From (14) and (15), it follows that

$$
x_{1} \frac{\partial c_{n}^{*}\left(\frac{\boldsymbol{x}}{1-\boldsymbol{x}}, r\right)}{\partial x_{1}}-x_{2} \frac{\partial c_{n}^{*}\left(\frac{\boldsymbol{x}}{1-\boldsymbol{x}}, r\right)}{\partial x_{2}}=c_{n}^{*}\left(\frac{\boldsymbol{x}}{1-\boldsymbol{x}}, r\right)\left(E_{1}+E_{2}\right),
$$

where

$$
\begin{aligned}
E_{1} & =\sum_{\substack{k+k_{3}+\ldots+k_{n}=r \\
k \neq 0}}\left(\frac{k x_{1}}{\left(1-x_{1}\right)^{2}\left(\frac{k x_{1}}{1-x_{1}}+\sum_{j=3}^{n} \frac{k_{j} x_{j}}{1-x_{j}}\right)}-\frac{k x_{2}}{\left(1-x_{2}\right)^{2}\left(\frac{k x_{2}}{1-x_{2}}+\sum_{j=3}^{n} \frac{k_{j} x_{j}}{1-x_{j}}\right)}\right) \\
& =k \sum_{\substack{k+k_{3}+\cdots+k_{n}=r \\
k \neq 0}} \frac{k x_{1} x_{2}\left(x_{1}-x_{2}\right)+\left(x_{1}-x_{2}\right)\left(1-x_{1} x_{2}\right) \sum_{j=3}^{n} \frac{k_{j} x_{j}}{1-x_{j}}}{\left(1-x_{1}\right)^{2}\left(\frac{k x_{1}}{1-x_{1}}+\sum_{j=3}^{n} \frac{k_{j} x_{j}}{1-x_{j}}\right)\left(1-x_{2}\right)^{2}\left(\frac{k x_{2}}{1-x_{2}}+\sum_{j=3}^{n} \frac{k_{j} x_{j}}{1-x_{j}}\right)}
\end{aligned}
$$

and

$$
\begin{aligned}
E_{2}= & \sum_{\substack{k+m+i_{3}+\cdots+i_{n}=r \\
k \neq 0, m \neq 0}}\left(\frac{k x_{1}}{\left(1-x_{1}\right)^{2}\left(\frac{k x_{1}}{1-x_{1}}+\frac{m x_{2}}{1-x_{2}}+\sum_{j=3}^{n} \frac{k_{j} x_{j}}{1-x_{j}}\right)}-\frac{k x_{2}}{\left(1-x_{2}\right)^{2}\left(\frac{k x_{2}}{1-x_{2}}+\frac{m x_{1}}{1-x_{1}}+\sum_{j=3}^{n} \frac{k_{j} x_{j}}{1-x_{j}}\right)}\right) \\
& =k \sum_{\substack{k+m+i_{3}+\cdots+i_{n}=r \\
k \neq 0, m \neq 0}} \frac{\delta_{2}}{\left(1-x_{1}\right)^{2}\left(\frac{k x_{1}}{1-x_{1}}+\frac{m x_{2}}{1-x_{2}}+\sum_{j=3}^{n} \frac{k_{j} x_{j}}{1-x_{j}}\right)\left(1-x_{2}\right)^{2}\left(\frac{k x_{2}}{1-x_{2}}+\frac{m x_{1}}{1-x_{1}}+\sum_{j=3}^{n} \frac{k_{j} x_{j}}{1-x_{j}}\right)}
\end{aligned}
$$

where

$$
\delta_{2}=k x_{1} x_{2}\left(x_{1}-x_{2}\right)+\left(\frac{\left(1-x_{2}\right)^{2} m x_{1}^{2}}{1-x_{1}}-\frac{\left(1-x_{1}\right)^{2} m x_{2}^{2}}{1-x_{2}}\right)+\left(x_{1}-x_{2}\right)\left(1-x_{1} x_{2}\right) \sum_{j=3}^{n} \frac{k_{j} x_{j}}{1-x_{j}} .
$$

Let $s(t)=\frac{(1-t)^{3}}{t^{2}}$. Then $s^{\prime}(t)=-\frac{t(2+t)(1-t)^{2}}{t^{4}} \leq 0$, this means that $s(t)$ is decreasing on $\mathbb{R}_{+}$, so $\frac{\left(1-x_{1}\right)^{3}}{x_{1}^{2}} \leq$ $\frac{\left(1-x_{2}\right)^{3}}{x_{2}^{2}}$, namely, $\frac{\left(1-x_{2}\right)^{2} m x_{1}^{2}}{1-x_{1}}-\frac{\left(1-x_{1}\right)^{2} m x_{2}^{2}}{1-x_{2}} \geq 0$. It is easy to see that $E_{1} \geq 0$ and $E_{2} \geq 0$ for $\boldsymbol{x} \in(0,1)^{n} \cup$ $(1,+\infty)^{n}$, so

$$
x_{1} \frac{\partial c_{n}^{*}\left(\frac{\boldsymbol{x}}{1-\boldsymbol{x}}, r\right)}{\partial x_{1}}-x_{2} \frac{\partial c_{n}^{*}\left(\frac{\boldsymbol{x}}{1-\boldsymbol{x}}, r\right)}{\partial x_{2}} \geq 0,
$$


By Lemma 3 , it follows that $c_{n}^{*}\left(\frac{\boldsymbol{x}}{1-\boldsymbol{x}}, r\right)$ is Schur-geometrically convex on $(0,1)^{n}$.

(ii) From (17) and combining with the Schur-geometrically convexity of $c_{n}^{*}\left(\frac{\boldsymbol{x}}{\boldsymbol{x}-1}, r\right)$ on $(1,+\infty)^{n}($ see Theorem 1), we can prove (ii) in Theorem 3.

The proof of Theorem 3 is completed.

\section{Proof of Theorem 4:}

For $r=1$ and $r=2$, it is easy to prove that $c_{n}^{*}\left(\frac{\boldsymbol{x}}{1-\boldsymbol{x}}, r\right)$ is Schur-harmonically convex on $(0,1)^{n}$.

Now consider the case of $r \geq 3$. By the symmetry of $c_{n}^{*}\left(\frac{x}{1-x}, r\right)$, without loss of generality, we can set $x_{1}>x_{2}$.

From (14) and (15), we have

$$
x_{1}^{2} \frac{\partial c_{n}^{*}\left(\frac{\boldsymbol{x}}{1-\boldsymbol{x}}, r\right)}{\partial x_{1}}-x_{2}^{2} \frac{\partial c_{n}^{*}\left(\frac{\boldsymbol{x}}{1-\boldsymbol{x}}, r\right)}{\partial x_{2}}=c_{n}^{*}\left(\frac{\boldsymbol{x}}{1-\boldsymbol{x}}, r\right)\left(F_{1}+F_{2}\right)
$$

where

$$
\begin{aligned}
F_{1} & =\sum_{\substack{k+k_{3}+\ldots+k_{n}=r \\
k \neq 0}}\left(\frac{k x_{1}^{2}}{\left(1-x_{1}\right)^{2}\left(\frac{k x_{1}}{1-x_{1}}+\sum_{j=3}^{n} \frac{k_{j} x_{j}}{1-x_{j}}\right)}-\frac{k x_{2}^{2}}{\left(1-x_{2}\right)^{2}\left(\frac{k x_{2}}{1-x_{2}}+\sum_{j=3}^{n} \frac{k_{j} x_{j}}{1-x_{j}}\right)}\right) \\
& =k \sum_{\substack{k+k_{3}+\ldots+k_{n}=r \\
k \neq 0}} \frac{k x_{1} x_{2}\left(x_{1}-x_{2}\right)+\left(x_{1}-x_{2}\right)\left(x_{1}+x_{2}-2 x_{1} x_{2}\right) \sum_{j=3}^{n} \frac{k_{j} x_{j}}{1-x_{j}}}{\left(1-x_{1}\right)^{2}\left(\frac{k x_{1}}{1-x_{1}}+\sum_{j=3}^{n} \frac{k_{j} x_{j}}{1-x_{j}}\right)\left(1-x_{2}\right)^{2}\left(\frac{k x_{2}}{1-x_{2}}+\sum_{j=3}^{n} \frac{k_{j} x_{j}}{1-x_{j}}\right)}
\end{aligned}
$$

and

$$
\begin{aligned}
F_{2}= & \sum_{\substack{k+m+i_{3}+\cdots+i_{n}=r \\
k \neq 0, m \neq 0}}\left(\frac{k x_{1}^{2}}{\left(1-x_{1}\right)^{2}\left(\frac{k x_{1}}{1-x_{1}}+\frac{m x_{2}}{1-x_{2}}+\sum_{j=3}^{n} \frac{k_{j} x_{j}}{1-x_{j}}\right)}-\frac{k x_{2}^{2}}{\left(1-x_{2}\right)^{2}\left(\frac{k x_{2}}{1-x_{2}}+\frac{m x_{1}}{1-x_{1}}+\sum_{j=3}^{n} \frac{k_{j} x_{j}}{1-x_{j}}\right)}\right) \\
& =k \sum_{\substack{k+m+i_{3}+\cdots+i_{n}=r \\
k \neq 0, m \neq 0}} \frac{\delta_{3}}{\left(1-x_{1}\right)^{2}\left(\frac{k x_{1}}{1-x_{1}}+\frac{m x_{2}}{1-x_{2}}+\sum_{j=3}^{n} \frac{k_{j} x_{j}}{1-x_{j}}\right)\left(1-x_{2}\right)^{2}\left(\frac{k x_{2}}{1-x_{2}}+\frac{m x_{1}}{1-x_{1}}+\sum_{j=3}^{n} \frac{k_{j} x_{j}}{1-x_{j}}\right)}
\end{aligned}
$$

where

$$
\delta_{3}=k x_{1} x_{2}\left(x_{1}-x_{2}\right)+\left(\frac{\left(1-x_{2}\right)^{2} m x_{1}^{3}}{1-x_{1}}-\frac{\left(1-x_{1}\right)^{2} m x_{2}^{3}}{1-x_{2}}\right)+\left(x_{1}-x_{2}\right)\left(x_{1}+x_{2}-2 x_{1} x_{2}\right) \sum_{j=3}^{n} \frac{k_{j} x_{j}}{1-x_{j}} .
$$

Let $v(t)=\frac{(1-t)^{3}}{m t^{3}}$. Then $v^{\prime}(t)=-\frac{3 m t^{2}(1-t)^{2}}{m^{2} t^{6}} \leq 0$ this means that $v(t)$ is decreasing on $\mathbb{R}$, so $\frac{\left(1-x_{1}\right)^{3}}{m x_{1}^{3}} \leq$ $\frac{\left(1-x_{2}\right)^{3}}{m x_{2}^{3}}$, namely, $\frac{\left(1-x_{2}\right)^{2} m x_{1}^{3}}{1-x_{1}}-\frac{\left(1-x_{1}\right)^{2} m x_{2}^{3}}{1-x_{2}} \geq 0$. It is easy to see that $F_{1} \geq 0$ and $F_{2} \geq 0$ for $\boldsymbol{x} \in(0,1)^{n}$, and then

$$
x_{1}^{2} \frac{\partial c_{n}^{*}\left(\frac{\boldsymbol{x}}{1-x}, r\right)}{\partial x_{1}}-x_{2}^{2} \frac{\partial c_{n}^{*}\left(\frac{\boldsymbol{x}}{1-x}, r\right)}{\partial x_{2}} \geq 0,
$$

By Lemma 3 , it follows that $c_{n}^{*}\left(\frac{\boldsymbol{x}}{1-\boldsymbol{x}}, r\right)$ is Schur-harmonically convex on $(0,1)^{n}$.

From Theorem 2, we know that $c_{n}^{*}\left(\frac{\boldsymbol{x}}{1-\boldsymbol{x}}, r\right)$ is Schur-geometrically convex on $(0,1)^{n}$, so that according to Lemma 5 , it follows that $c_{n}^{*}\left(\frac{\boldsymbol{x}}{1-\boldsymbol{x}}, r\right)$ is Schur-harmonically convex on $(0,1)^{n}$. 
(ii) From 17) and combining with the Schur-harmonically convexity of $c_{n}^{*}\left(\frac{\boldsymbol{x}}{\boldsymbol{x}-1}, r\right)$ on $(1,+\infty)^{n}$ (see Theorem 1), we can prove (ii) in Theorem 4.

The proof of Theorem 4 is completed.

Here, a question arises naturally.

Question 1. For $\boldsymbol{x} \in\left(0, \frac{1}{2}\right)^{n}$, what is the Schur-convexity of $c_{n}^{*}\left(\frac{\boldsymbol{x}}{1-\boldsymbol{x}}, r\right)$ ?

\section{Applications}

It is not difficult to prove the following result by applying Theorem 2 and the majorizing relation

$$
\left(A_{n}(\boldsymbol{x}), A_{n}(\boldsymbol{x}), \ldots, A_{n}(\boldsymbol{x})\right) \prec\left(x_{1}, x_{2}, \ldots, x_{n}\right) .
$$

Theorem 5. If $\boldsymbol{x}=\left(x_{1}, x_{2}, \ldots, x_{n}\right) \in\left[\frac{1}{2}, 1\right)^{n}$ and $r \in \mathbb{N}$, or $r$ is even integer and $\boldsymbol{x} \in(1,+\infty)^{n}$ or $r$ is odd integer and $\boldsymbol{x} \in \mathbb{R}_{-}^{n}$, then

$$
c_{n}^{*}\left(\frac{\boldsymbol{x}}{1-\boldsymbol{x}}, r\right) \geq\left(\frac{r A_{n}(\boldsymbol{x})}{1-A_{n}(\boldsymbol{x})}\right)^{\left(\begin{array}{c}
n+r-1 \\
r
\end{array}\right)},
$$

where $A_{n}(\boldsymbol{x})=\frac{1}{n} \sum_{i=1}^{n} x_{i}$ and $\left(\begin{array}{c}n+r-1 \\ r\end{array}\right)=\frac{(n+r-1) !}{r !((n+r-1)-r) !}$.

If $r$ is odd and $\boldsymbol{x} \in(1,+\infty)^{n}$, or $r$ is even integer and $\boldsymbol{x} \in \mathbb{R}_{-}^{n}$, then the inequality (18) is reversed.

By Theorem 3 and the majorizing relation

$$
\left(\log G_{n}(\boldsymbol{x}), \log G_{n}(\boldsymbol{x}), \ldots, \log G_{n}(\boldsymbol{x})\right) \prec\left(\log x_{1}, \log x_{2}, \ldots, \log x_{n}\right),
$$

we can establish the following theorem.

Theorem 6. If $\boldsymbol{x}=\left(x_{1}, x_{2}, \ldots, x_{n}\right) \in(0,1)^{n}$ and $r \in \mathbb{N}$ or $r$ is even integer $\boldsymbol{x} \in(1,+\infty)^{n}$, then

$$
c_{n}^{*}\left(\frac{\boldsymbol{x}}{1-\boldsymbol{x}}, r\right) \geq\left(\frac{r G_{n}(\boldsymbol{x})}{1-G_{n}(\boldsymbol{x})}\right)^{\left(\begin{array}{c}
n+r-1 \\
r
\end{array}\right)},
$$

where $G_{n}(\boldsymbol{x})=\sqrt[n]{\prod_{i=1}^{n} x_{i}}$ and $\left(\begin{array}{c}n+r-1 \\ r\end{array}\right)=\frac{(n+r-1) !}{r !((n+r-1)-r) !}$.

If $r$ is odd integer and $\boldsymbol{x} \in(1,+\infty)^{n}$, then the inequality (19) is reversed.

By using Theorem 4 and the majorizing relation

$$
\left(\frac{1}{H_{n}(\boldsymbol{x})}, \frac{1}{H_{n}(\boldsymbol{x})}, \ldots, \frac{1}{H_{n}(\boldsymbol{x})}\right) \prec\left(\frac{1}{x_{1}}, \frac{1}{x_{2}}, \ldots, \frac{1}{x_{n}}\right),
$$

we obtain the following theorem. 
Theorem 7. If $\boldsymbol{x}=\left(x_{1}, x_{2}, \ldots, x_{n}\right) \in(0,1)^{n}$ and $r \in \mathbb{N}$, or $r$ is even integer and $\boldsymbol{x} \in(1,+\infty)^{n}$, then

$$
c_{n}^{*}\left(\frac{\boldsymbol{x}}{1-\boldsymbol{x}}, r\right) \geq\left(\frac{r H_{n}(\boldsymbol{x})}{1-H_{n}(\boldsymbol{x})}\right)^{\left(\begin{array}{c}
n+r-1 \\
r
\end{array}\right)},
$$

where $H_{n}(\boldsymbol{x})=\frac{n}{\sum_{i=1}^{n} x_{i}^{-1}}$ and $\left(\begin{array}{c}n+r-1 \\ r\end{array}\right)=\frac{(n+r-1) !}{r !((n+r-1)-r) !}$.

If $r$ is odd and $\boldsymbol{x} \in\left(1,+\infty^{n}\right.$, then the inequality (20) is reversed.

By applying Theorem 2 and Lemma 6, it is not difficult to show the following theorem.

Theorem 8. If $\boldsymbol{x}=\left(x_{1}, x_{2}, \ldots, x_{n}\right) \in \mathbb{R}_{+}^{n}, n \geq 2$ and $k \in \mathbb{N}, 0<r \leq s$, then

$$
\prod_{i_{1}+i_{2}+\cdots+i_{n}=k} \sum_{j=1}^{n} \frac{i_{j} x_{j}^{r}}{\sum_{j=1}^{n} x_{j}^{r}-x_{j}^{r}} \leq \prod_{i_{1}+i_{2}+\cdots+i_{n}=k} \sum_{j=1}^{n} \frac{i_{j} x_{j}^{s}}{\sum_{j=1}^{n} x_{j}^{s}-x_{j}^{s}} .
$$

By Theorem 2 and Lemma 7, we establish the following theorem.

Theorem 9. Let $\boldsymbol{x}=\left(x_{1}, x_{2}, \ldots, x_{n}\right) \in \mathbb{R}_{+}^{n}, n \geq 2, \sum_{i=1}^{n} x_{i}=s>0, c \geq s$. Then

$$
\prod_{i_{1}+i_{2}+\cdots+i_{n}=k} \sum_{j=1}^{n} \frac{i_{j}\left(c-x_{j}\right)}{(n-1) c-\left(s-x_{i}\right)} \leq \prod_{i_{1}+i_{2}+\cdots+i_{n}=k} \sum_{j=1}^{n} \frac{i_{j} x_{j}}{s-x_{j}} .
$$

Discovering and judging Schur convexity of various symmetric functions is an important subject in the study of the majorization theory. In recent years, many domestic scholars have made a lot of achievements in this field (see monographs [27, 28]).

\section{References}

[1] A. W. Marshall, I. Olkin, and B. C. Arnold, Inequalities: Theory of Majorization and Its Application (Second Edition), Springer, New York, 2011.

[2] B. Y. Wang, Foundations of Majorization Inequalities, Beijing Normal University Press, Beijing, 1990. (in Chinese)

[3] X. M. Zhang, Geometrically Convex Functions, An'hui University Press, Hefei, 2004. (in Chinese)

[4] Y. M. Chu, X. M. Zhang, and G. D. Wang, The Schur geometrical convexity of the extended mean values, Journal of Convex Analysis, 2008, 15(4), 707-718.

[5] K. Z. Guan, A class of symmetric functions for multiplicatively convex function, Mathematical Inequalities \& Applications, 2007, 10(4), 745-753.

[6] T.-C. Sun, Y.-P. Lv, and Y.-M. Chu, Schur multiplicative and harmonic convexities of generalized Heronian mean in $n$ variables and their applications, International Journal of Pure and Applied Mathematics, 2009, 55(1), 25-33.

[7] Y. M. Chu, and T. C. Sun, The Schur harmonic convexity for a class of symmetric functions, Acta Mathematica Scientia, 2010, 30B(5), 1501-1506.

[8] Y.-M. Chu, G.-D.Wang, and X.-H. Zhang, The Schur multiplicative and harmonic convexities of the complete symmetric function, Mathematische Nachrichten, 2011, 284(5-6), 653-663.

[9] Y.-M. Chu, and Y.-P. Lv, The Schur harmonic convexity of the Hamy symmetric function and its applications, Journal of Inequalities and Applications, 2009, Article ID 838529, 10 pages.

[10] W. F. Xia, and Y. M. Chu, Schur-convexity for a class of symmetric functions and its applications, Journal of Inequalities and Applications, 2009, Article ID 493759, 15 pages.

[11] K.-Z. Guan, Schur-convexity of the complete symmetric function, Mathematical Inequalities \& Applications, 2006, 9(4), $567-576$.

[12] M. B. Sun, N. B. Chen, and S. H. Li, Some properties of a class of symmetric functions and its applications, Mathematische Nachrichten, 2014, doi: 10.1002/mana.201300073.

[13] W.-F. Xia, and Y.-M. Chu, Schur convexity and Schur multiplicative convexity for a class of symmeric functions with applications, Ukrainian Mathematical Journal, 2009, 61(10), 1541-1555. 
[14] Ionel Rovenţa, Schur convexity of a class of symmetric functions, Annals of the University of Craiova, Mathematics and Computer Science Series, 2010, 37(1), 12-18.

[15] W.-F. Xia, and Y.-M. Chu, On Schur convexity of some symmetric functions, Journal of Inequalities and Applications, 2010, Article ID 543250, 12 pages.

[16] J.-X. Meng, Y.-M. Chu, and X.-M. Tang, The Schur-harmonic-convexity of dual form of the Hamy symmetric function, Matematiqki Vesnik, 2010, 62(1), 37-46.

[17] Y.-M. Chu, W.-F. Xia, and T.-H. Zhao, Some properties for a class of symmetric functions and applications, Journal of Mathematical Inequalities, 2011, 5(1), 1-11.

[18] K.-Z. Guan, and R.-K. Guan, Some properties of a generalized Hamy symmetric function and its applications, Journal of Mathematical Analysis and Applications, 2011, 376, 494-505.

[19] W.-M. Qian, Schur convexity for the ratios of the Hamy and generalized Hamy symmetric functions, Journal of Inequalities and Applications, 2011, 2011:131, doi:10.1186/1029-242X-2011-131.

[20] Y.-M. Chu, W.-F. Xia, and X.-H. Zhang, The Schur concavity, Schur multiplicative and harmonic convexities of the second dual form of the Hamy symmetric function with applications, Journal of Multivariate Analysis, 2012, 105(1), 412-421.

[21] Ionel Rovenţa, A note on Schur-concave functions, Journal of Inequalities and Applications, 2012, 2012:159, doi:10.1186/1029-242X-2012-159.

[22] W.-F. Xia, X.-H. Zhang, G.-D. Wang and Y.-M. Chu, Some properties for a class of symmetric functions with applications, Indian J. Pure Appl. Math., 2012, 43(3), 227-249.

[23] H.-N. Shi and J. Zhang, Schur-convexity of dual form of some symmetric functions, Journal of Inequalities and Applications, 2013, 2013,295, doi:10.1186/1029-242X-2013-295.

[24] K.-S. Zhang, and H.-N. Shi, Schur convexity of dual form of the complete symmetric function, Mathematical Inequalities \& Applications, 2013, 16(4), 963-970.

[25] H.-N. Shi, J. Zhang and Q.-H. Ma. Schur-convexity, Schur-geometric and Schur-harmonic convexity for a composite function of complete symmetric function, SpringerPlus (2016) 5,296.

[26] X.-H. Zhang and Y.-M. Chu, New discussion to analytic Inequalities, Harbin, Harbin Institute of Technology Press, 2009.(in Chinese)

[27] H.-N. Shi. Majorization Theory and Analytical Inequalities, Harbin: Harbin Institute of Technology Press, 2012.(in Chinese)

[28] H.-N. Shi. Schur-Convex Functions and Inequalities, Harbin: Harbin Institute of Technology Press, 2012.(in Chinese) 